\title{
Multiscale Mixing Efficiencies for Steady Sources
}

\author{
Charles R. Doering* \\ Department of Mathematics and Michigan Center for Theoretical Physics, \\ University of Michigan, Ann Arbor, MI 48109-1043, USA
}

Jean-Luc Thiffeault ${ }^{\dagger}$

Department of Mathematics, Imperial College London, SW7 2AZ, United Kingdom

\begin{abstract}
Multiscale mixing efficiencies for passive scalar advection are defined in terms of the suppression of variance weighted at various length scales. We consider scalars maintained by temporally steady but spatially inhomogeneous sources, stirred by statistically homogeneous and isotropic incompressible flows including fully developed turbulence. The mixing efficiencies are rigorously bounded in terms of the Péclet number and specific quantitative features of the source. Scaling exponents for the bounds at high Péclet number depend on the spectrum of length scales in the source, indicating that molecular diffusion plays a more important quantitative role than that implied by classical eddy diffusion theories.
\end{abstract}

PACS numbers: 47.27.Qb, 92.10.Lq, 92.60.Ek, 94.10.Lf

Keywords: advection, convection, diffusion, mixing, turbulent diffusion

Introduction. Mixing in fluid flows plays a central role in many scientific and engineering applications and is the subject of a large body of theoretical research [1]. In this paper we consider the mixing of a passive scalar maintained by a temporally steady but spatially inhomogeneous source without a clear separation of length scales between the source and the stirrer, as is necessary for multiscale analyses, or when length scales in the source are the smallest scales in the problem.

The effectiveness of a given stirring velocity vector field in a statistically stationary state is naturally gauged by its ability to suppress the space-time averaged variance of the scalar: a decrease in the variance indicates a more uniformly mixed scalar. The ratio of the scalar variance in the presence of stirring to that resulting from molecular diffusion alone defines a dimensionless measure of the mixing efficiency of the flow. Scalar variances may be weighted at diverse spatial length scales, so we introduce a family of mixing efficiencies that measure the effectiveness of the stirring on different scales. Not unexpectedly, these mixing efficiencies generally depend on details of both the source and the stirring. Sawford \& Hunt [2] highlighted the role of source size in particle dispersion models for the case of small sources, showing that the concentration variance depends explictly on the molecular diffusivity. Here we make direct use of the advection-diffusion equation to show the nature of this dependence for a wide range of source types.

For statistically stationary, homogeneous and isotropic flows - including turbulence - we derive new, mathematically rigorous and physically relevant limits on these ef-

\footnotetext{
*Electronic address: doering@umich.edu

${ }^{\dagger}$ Electronic address: jeanluc@imperial.ac.uk
}

ficiencies in terms of the Péclet number and quantitative features of the source alone. We discover that the highPéclet number scaling exponents of the efficiency bounds depend on the spatial dimension, the length scales upon which the variance is observed, and spatial scales in the source. These results show that the spectrum of length scales in the source may be more important than the distribution of length scales in the flow in determining the mixing efficiencies. Moreover, we derive novel quantitative estimates of nontrivial scaling exponents for the mixing efficiencies in cases where the source is fractal or measure-valued. These are anomalous scalings that cannot be deduced from dimensional arguments alone.

Problem statement and definitions. The advectiondiffusion equation for the concentration $\theta(\boldsymbol{x}, t)$ of a passive scalar is

$$
\partial_{t} \theta+\boldsymbol{u} \cdot \nabla \theta=\kappa \Delta \theta+s
$$

where $\kappa$ is the molecular diffusivity. We restrict attention to spatially periodic boundary conditions for $\boldsymbol{x} \in[0, L]^{d}$. The time-independent source $s(\boldsymbol{x})$ in (1) is taken (without loss of generality) to have spatial mean zero so that eventually the concentration $\theta(\boldsymbol{x}, t)$ will have spatial mean zero as well.

The velocity field $\boldsymbol{u}(\boldsymbol{x}, t)$ is given. It could be the solution of some dynamical equations or a particular stochastic process, but in any case we consider it to be a prescribed divergence-free vector field with the following equal-time single-point statistical properties shared by homogeneous isotropic turbulence:

$$
\begin{aligned}
& \overline{u_{i}(\boldsymbol{x}, \cdot)}=0, \quad \overline{u_{i}(\boldsymbol{x}, \cdot) u_{j}(\boldsymbol{x}, \cdot)}=\frac{U^{2}}{d} \delta_{i j} \\
& \overline{u_{i}(\boldsymbol{x}, \cdot) \frac{\partial u_{j}(\boldsymbol{x}, \cdot)}{\partial x_{k}}}=0, \quad \overline{\frac{\partial u_{i}(\boldsymbol{x}, \cdot)}{\partial x_{k}} \frac{\partial u_{j}(\boldsymbol{x}, \cdot)}{\partial x_{k}}}=\frac{\Omega^{2}}{d} \delta_{i j}
\end{aligned}
$$


where overbar represents the long-time average (assumed to exist) at each point in space. The r.m.s. velocity $U$ measures the strength of the stirring and $\Omega$ indicates the flow field's strain or shear content. The ratio $\lambda=U / \Omega$ corresponds to the Taylor microscale for homogeneous isotropic turbulence. The Péclet number for the flow is $\mathrm{Pe}=U L / \kappa$.

We quantify the mixing of the scalar by the magnitude of the variances $\left\langle|\nabla \theta|^{2}\right\rangle,\left\langle\theta^{2}\right\rangle$, and $\left\langle\left|\nabla^{-1} \theta\right|^{2}\right\rangle$ where $\langle\cdot\rangle$ denotes space-time averaging. (The operator $\nabla^{-1}$ is defined by its Fourier representation in the periodic domain, $\nabla^{-1} \rightarrow-\mathrm{i} \boldsymbol{k} / k^{2}$.) These variances measure the fluctuations of $\theta$ at relatively small, intermediate and large length scales. Collectively we write the norms $\left\langle\left|\nabla^{p} \theta\right|^{2}\right\rangle$ for $p=1,0$, and -1 . Note that $\left\langle\left|\nabla^{-1} \theta\right|^{2}\right\rangle$ is reminiscent of the recently-introduced "mix-norm" [3], as both downplay the importance of the small scales.

In order to define dimensionless mixing efficiencies we use the baseline variances defined by the solution $\theta_{0}$ of equation (1) with the same source but $\boldsymbol{u}=0$, i.e., $\theta_{0}=-\kappa^{-1} \Delta^{-1} s$. Comparing fluctuations in the presence of stirring to the moments $\left\langle\left|\nabla^{p} \theta_{0}\right|^{2}\right\rangle$ allows us to gauge the effect of stirring. We define the dimensionless mixing efficiencies $\mathcal{E}_{p}$ by

$$
\mathcal{E}_{p}^{2}:=\left\langle\left|\nabla^{p} \theta_{0}\right|^{2}\right\rangle /\left\langle\left|\nabla^{p} \theta\right|^{2}\right\rangle
$$

These efficiencies increase when the stirring decreases the scalar variances relative to molecular diffusion alone.

Mixing efficiency bounds. Upper bounds on $\mathcal{E}_{p}$ result from lower limits on the variances. We begin with estimates on $\left\langle\theta^{2}\right\rangle$ using the method developed in [4]: multiply (1) by an arbitrary (but smooth, spatially periodic) function $\varphi(\boldsymbol{x})$, average, and integrate by parts to find

$$
\langle\theta(\boldsymbol{u} \cdot \nabla+\kappa \Delta) \varphi\rangle=-\langle\varphi s\rangle .
$$

Fluctuations are bounded from below via the min-max variational expression

$$
\left\langle\theta^{2}\right\rangle \geq \max _{\varphi} \min _{\vartheta}\left\{\left\langle\vartheta^{2}\right\rangle \mid\langle\vartheta(\boldsymbol{u} \cdot \nabla \varphi+\kappa \Delta \varphi)\rangle=-\langle\varphi s\rangle\right\} .
$$

The minimization over $\vartheta$ is easily achieved, equivalent to application of the Cauchy-Schwarz inequality, yielding

$$
\left\langle\theta^{2}\right\rangle \geq \max _{\varphi}\langle\varphi s\rangle^{2} /\left\langle(\boldsymbol{u} \cdot \nabla \varphi+\kappa \Delta \varphi)^{2}\right\rangle .
$$

This is the sort of variational estimate derived in [4]. Plasting and Young recently enhanced that analysis by including the scalar dissipation rate as a constraint [5].

The subsequent maximization over $\varphi$ is particularly simple for statistically homogeneous and isotropic flows satisfying (2), for then the denominator in (5) is

$$
\left\langle(\boldsymbol{u} \cdot \nabla \varphi+\kappa \Delta \varphi)^{2}\right\rangle=\left\langle\frac{U^{2}}{d}|\nabla \varphi|^{2}+\kappa^{2}(\Delta \varphi)^{2}\right\rangle,
$$

i.e., the quadratic form $\left\langle\varphi\left[\kappa^{2} \Delta^{2}-\left(U^{2} / d\right) \Delta\right] \varphi\right\rangle$. Hence the variational problem (5) yields

$$
\mathcal{E}_{0}^{2} \leq \frac{\left\langle s \Delta^{-2} s\right\rangle}{\left\langle s\left\{\Delta^{2}-\left(U^{2} / \kappa^{2} d\right) \Delta\right\}^{-1} s\right\rangle},
$$

a bound that depends on the "shape" of the source function but not its amplitude, and on the stirring velocity field only through its influence on the length scale $\kappa / U=\mathrm{Pe}^{-1} L$.

Limits for the small scale and large scale efficiencies $\mathcal{E}_{ \pm 1}$ are obtained from (4) in the same manner after integrations by parts and application of the Cauchy-Schwarz inequality. For the gradient variance

$$
\langle\varphi s\rangle^{2}=\langle(\boldsymbol{u} \varphi+\kappa \nabla \varphi) \cdot \nabla \theta\rangle^{2} \leq\left\langle|\boldsymbol{u} \varphi+\kappa \nabla \varphi|^{2}\right\rangle\left\langle|\nabla \theta|^{2}\right\rangle
$$

So

$$
\left\langle|\nabla \theta|^{2}\right\rangle \geq \max _{\varphi}\langle\varphi s\rangle^{2} /\left\langle(\boldsymbol{u} \varphi+\kappa \nabla \varphi)^{2}\right\rangle
$$

A potentially sharper bound involving the full two-point correlation function for the velocity field can be obtained by formally minimizing over $\theta[6]$, but for our purposes the estimate (8) suffices. For statistically homogeneous isotropic flows the denominator above is $\left\langle\varphi\left[-\kappa^{2} \Delta+U^{2}\right] \varphi\right\rangle$ and optimization over $\varphi$ leads to

$$
\mathcal{E}_{1}^{2} \leq \frac{\left\langle s\left(-\Delta^{-1}\right) s\right\rangle}{\left\langle s\left\{-\Delta+U^{2} / \kappa^{2}\right\}^{-1} s\right\rangle} .
$$

The bound on large scale fluctuations follows from (4) using $\theta=\nabla \cdot \nabla^{-1} \theta$, an integration by parts, and CauchySchwarz:

$$
\begin{aligned}
& \langle\varphi s\rangle^{2}=\left\langle\nabla(\boldsymbol{u} \cdot \nabla \varphi+\kappa \Delta \varphi) \cdot\left(\nabla^{-1} \theta\right)\right\rangle^{2} \\
& \quad \leq\left\langle|\nabla \boldsymbol{u} \cdot \nabla \varphi+\boldsymbol{u} \cdot \nabla \nabla \varphi+\kappa \Delta \nabla \varphi|^{2}\right\rangle\left\langle\left|\nabla^{-1} \theta\right|^{2}\right\rangle
\end{aligned}
$$

so that

$$
\left\langle\left|\nabla^{-1} \theta\right|^{2}\right\rangle \geq \max _{\varphi} \frac{\langle\varphi s\rangle^{2}}{\left\langle|\nabla \boldsymbol{u} \cdot \nabla \varphi+\boldsymbol{u} \cdot \nabla \nabla \varphi+\kappa \Delta \nabla \varphi|^{2}\right\rangle} .
$$

For statistically homogeneous isotropic flows the denominator is $\left\langle\varphi\left[-\kappa^{2} \Delta^{3}+\left(U^{2} / d\right) \Delta^{2}-\left(\Omega^{2} / d\right) \Delta\right] \varphi\right\rangle$ so that

$$
\mathcal{E}_{-1}^{2} \leq \frac{\left\langle s\left(-\Delta^{-3}\right) s\right\rangle}{\left\langle s\left\{-\Delta^{3}+\left(U^{2} / \kappa^{2} d\right) \Delta^{2}-\left(\Omega^{2} / \kappa^{2} d\right) \Delta\right\}^{-1} s\right\rangle} .
$$

It is helpful to rewrite the bounds in Fourier space:

$$
\begin{gathered}
\mathcal{E}_{1}^{2} \leq \frac{\sum_{\boldsymbol{k}}\left|s_{\boldsymbol{k}}\right|^{2} / k^{2}}{\sum_{\boldsymbol{k}}\left|s_{\boldsymbol{k}}\right|^{2} /\left(k^{2}+\mathrm{Pe}^{2}\right)}, \\
\mathcal{E}_{0}^{2} \leq \frac{\sum_{\boldsymbol{k}}\left|s_{\boldsymbol{k}}\right|^{2} / k^{4}}{\sum_{\boldsymbol{k}}\left|s_{\boldsymbol{k}}\right|^{2} /\left(k^{4}+\mathrm{Pe}^{2} k^{2} / d\right)}, \\
\mathcal{E}_{-1}^{2} \leq \frac{\sum_{\boldsymbol{k}}\left|s_{\boldsymbol{k}}\right|^{2} / k^{6}}{\sum_{\boldsymbol{k}}\left|s_{\boldsymbol{k}}\right|^{2} /\left(k^{6}+\mathrm{Pe}^{2} k^{4} / d+\mathrm{Pe}^{2} k^{2} / \lambda^{2} d\right)}
\end{gathered}
$$

where we have rescaled $[0, L]^{d}$ to $[0,1]^{d}$ so that wavevector components are integer multiples of $2 \pi$. Now we investigate the large Péclet number behavior of these bounds for a variety of classes of sources. 
Monochromatic Sources. For sources that depend only on a single wavenumber $k_{s}$, the bounds simplify to

$$
\begin{gathered}
\mathcal{E}_{1} \leq \sqrt{1+\mathrm{Pe}^{2} / k_{s}^{2}}, \\
\mathcal{E}_{0} \leq \sqrt{1+\mathrm{Pe}^{2} / k_{s}^{2} d}, \\
\mathcal{E}_{-1} \leq \sqrt{1+\mathrm{Pe}^{2} / k_{s}^{2} d+\mathrm{Pe}^{2} / \lambda^{2} k_{s}^{4} d} .
\end{gathered}
$$

Each efficiency is asymptotically proportional to Pe, corresponding to the expected suppression of variance if the molecular diffusivity $\kappa$ is replaced by an eddy diffusivity proportional to $U L$. Moreover these upper bounds are sharp: they may be realized by uniform flow fields whose direction varies appropriately in time to satisfy the weak statistical homogeneity and isotropy conditions used in the analysis [5, 6]. Each estimate also exhibits a decreasing dependence on the length scale of the source: at high Pe the estimates for the small and intermediate scale efficiencies $\mathcal{E}_{1}$ and $\mathcal{E}_{0}$ are $\sim \mathrm{Pe} / k_{s}$. This suggests that an eddy diffusivity might better be defined as a product of $U$ and a length scale $\sim k_{s}^{-1}$ characterizing the source, rather than some scale characterizing the stirring.

Square Integrable Sources and Sinks. In situations where the Fourier coefficients are square-summable, the asymptotic $\mathrm{Pe} \rightarrow \infty$ behavior of the mixing efficiency bounds for smooth sources is straightforward:

$$
\begin{gathered}
\mathcal{E}_{1} \leq \operatorname{Pe} \sqrt{\frac{\sum_{\boldsymbol{k}}\left|s_{\boldsymbol{k}}\right|^{2} / k^{2}}{\sum_{\boldsymbol{k}}\left|s_{\boldsymbol{k}}\right|^{2}}}, \\
\mathcal{E}_{0} \leq \operatorname{Pe} \sqrt{\frac{\sum_{\boldsymbol{k}}\left|s_{\boldsymbol{k}}\right|^{2} / k^{4}}{d \sum_{\boldsymbol{k}}\left|s_{\boldsymbol{k}}\right|^{2} / k^{2}}}, \\
\mathcal{E}_{-1} \leq \mathrm{Pe} \sqrt{\frac{\sum_{\boldsymbol{k}}\left|s_{\boldsymbol{k}}\right|^{2} / k^{6}}{d \sum_{\boldsymbol{k}}\left|s_{\boldsymbol{k}}\right|^{2} /\left(k^{4}+k^{2} / \lambda^{2}\right)}} .
\end{gathered}
$$

These are the same Pe scalings as observed for monochromatic sources but with prefactors involving distinct characteristic length scales of the source tailored to the scales where the different efficiencies are tuned. We reiterate that the $\mathrm{Pe}^{1}$ scaling of the efficiencies is precisely that which is expected from the conventional notion of eddy diffusion, at least with regard to the $U$ and $\kappa$ dependence. A novel feature of these rigorous estimates is that the "mixing lengths" to be employed in constructing the effective diffusion from $U$ depend on (i) the source structure rather than some characteristic persistence length in the flow, and (ii) the length scales in the concentration fluctuations stressed by the different multiscale efficiencies.

Rough Sources. The Péclet number scaling may actually change for "rough" sources, i.e., when $s(\boldsymbol{x})$ is not square integrable, resulting in anomalous behavior for some of the efficiencies. The roughest physically meaningful sources are measure-valued sources like $\delta$-functions with nondecaying Fourier coefficients $\left|s_{\boldsymbol{k}}\right|=\mathcal{O}(1)$ as $k \rightarrow$ $\infty$. Then the sums in (11a) and the denominator of (11b) diverge in $d=2$ or 3 so those scalings are invalid. In this case the Pe dependence of $\mathcal{E}_{1}$ disappears altogether and all finite kinetic energy stirring fields are completely ineffective at suppressing small scale fluctuations.

To determine the high-Pe behavior of $\mathcal{E}_{0}$ we approximate sums by integrals. The denominator of $(10 \mathrm{~b})$ is

$$
\sum_{\boldsymbol{k}} \frac{1}{k^{4}+\left(\mathrm{Pe}^{2} / d\right) k^{2}} \sim \int_{2 \pi}^{\infty} \frac{k^{d-1} \mathrm{~d} k}{k^{4}+\mathrm{Pe}^{2} k^{2} /\left(4 \pi^{2} d\right)} .
$$

For $d=2$ the integral in (12) is

$$
\int_{2 \pi}^{\infty} \frac{k \mathrm{~d} k}{k^{4}+\mathrm{Pe}^{2} k^{2} / 8 \pi^{2}} \sim \frac{\log \mathrm{Pe}}{\mathrm{Pe}^{2}},
$$

resulting in the asymptotic bound

$$
\mathcal{E}_{0} \lesssim \mathrm{Pe} / \sqrt{\log \mathrm{Pe}}, \quad d=2 .
$$

Hence there is at the very least a logarithmic correction to $\mathcal{E}_{0}$ as compared to the square integrable source case.

For $d=3$ the integral in (12) becomes

$$
\int_{2 \pi}^{\infty} \frac{k^{2} \mathrm{~d} k}{k^{4}+\mathrm{Pe}^{2} k^{2} / 12 \pi^{2}} \sim \frac{1}{\mathrm{Pe}}
$$

resulting in an anomalous scaling bound

$$
\mathcal{E}_{0} \lesssim \sqrt{\mathrm{Pe}}, \quad d=3 .
$$

This is a dramatic modification of the classical scaling.

Similar analysis shows that the upper bound on the large scale mixing efficiency $\mathcal{E}_{-1} \sim$ Pe in (11c) persists even for these roughest sources.

Rough sources with a cutoff. Approximate delta-like sources of small but finite size $\ell$ have Fourier coefficients $s_{\boldsymbol{k}}$ that are approximately constant in magnitude up to a cutoff wavenumber of order $2 \pi / \ell$, beyond which the spectrum decays as for a smooth source. We may deduce the behavior of the bound on $\mathcal{E}_{0}$ for such sources by inserting an upper limit at $L / \ell \gg 1$ into the integral in (12). For large but intermediate Péclet numbers satisfying $1 \ll \mathrm{Pe} \ll L / \ell$, the cutoff is ineffective so the logarithmic correction (14) in $d=2$ and the anomalous scaling (16) in $d=3$ appear. However for $\mathrm{Pe} \gg L / \ell$, i.e., when the modified Péclet number based on the smallest scale in the source $U \ell / \kappa \gg 1$, the smooth source results apply and we recover the mixing efficiency bounds linear in Pe, as in (11). Figure (1) shows this scaling transition for the $d=3$ case. Even in the ultimate regime where the source appears smooth, the prefactor in front of the high-Pe scaling bounds are significantly diminished by the small scales in the source: $\mathcal{E}_{0} \lesssim[\log (L / \ell)]^{-1 / 2} \mathrm{Pe}$ in $d=2$, and $\mathcal{E}_{0} \lesssim[\ell / L]^{1 / 2} \mathrm{Pe}$ in $d=3$.

Fractal Sources. We may also analyze anomalous scalings for more general "fractal" rough sources where the Fourier spectrum $\left|s_{\boldsymbol{k}}\right|$ decays as $k^{-\gamma}$ with $0 \leq \gamma \leq d / 2$. The roughest measure-valued sources have $\gamma=0$ while 


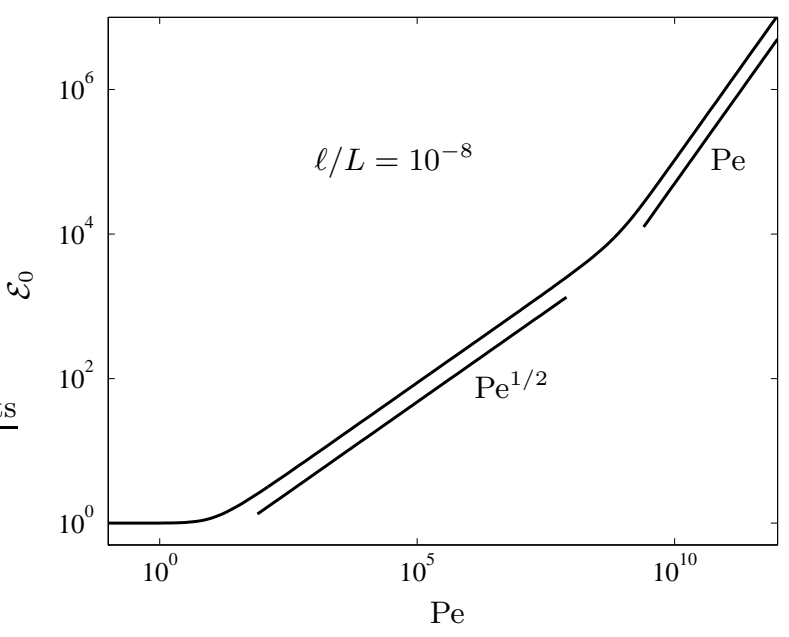

FIG. 1: Upper bound for the mixing efficiency $\mathcal{E}_{0}$ as a function of Péclet number for a small source with $\ell=10^{-8} \mathrm{~L}$ stirred by a three-dimensional statistically homogeneous and isotropic flow [computed from Eq. (10b)]. The intermediate $\mathrm{Pe}^{1 / 2}$ scaling for $1 \ll \mathrm{Pe} \ll(L / \ell)$ is evident.

for $\gamma>d / 2$ the source is square integrable and thus effectively smooth as far as these multiscale mixing efficiencies are concerned. In order to examine the high Péclet number asymptotics of the bounds on the various $\mathcal{E}_{p}$ we estimate integrals similar to (12) but with an extra factor of $k^{-2 \gamma}$ in the numerator arising from $\left|s_{\boldsymbol{k}}\right|^{2}$. The results are summarized in Table I. In $d=2$ the scaling for $\mathcal{E}_{1}$ is anomalous for any degree of roughness while $\mathcal{E}_{0}$ is anomalous only for $\gamma=0$. In $d=3, \mathcal{E}_{1}$ is again anomalous for any degree of roughness while $\mathcal{E}_{0}$ scales anomalously for $0 \leq \gamma<1 / 2$. For both $d=2$ and 3 the bound on the large scale mixing efficiency $\mathcal{E}_{-1}$ is always conventional. Of course these scalings neglect any large- $k$ cutoff for the rough sources. If there is a cutoff at wavenumber $2 \pi / \ell$ then the same arguments apply to recover the normal scaling $\sim \mathrm{Pe}$ for $\mathrm{Pe} \gg L / \ell$.

Discussion. The multiscale mixing efficiency bounds derived here reveal new aspects of the ability - and in some cases the inability of statistically homogeneous and isotropic incompressible flows to effectively suppress fluctuations in passive scalars. Several notable features of the phenomena have emerged from this analysis. One is that the structure of the source maintaining the scalar concentration plays a central role in efficiency of mixing at different length scales while the detailed structure of the flow, i.e., the spectrum of length (and time) scales in the velocity field, plays only a secondary role as far as the small and intermediate scale efficiencies $\mathcal{E}_{1}$ and $\mathcal{E}_{0}$ are concerned. The bound on $\mathcal{E}_{-1}$ in (11c), however, means that small scale structure and strain in the advecting flow could possibly enhance the large scale mixing efficiency.

For rough sources the efficiencies scale at least as anomalously as indicated in Table I, and no clever small scale stirring can alleviate this effect. When efficiency bounds scale anomalously, molecular diffusion plays a much more important role than implied by conventional eddy diffusion theories. For then - as far as variance sup-

TABLE I: Scalings of the bound on the mixing efficiency $\mathcal{E}_{p}$ as functions of the source roughness exponent $\gamma$ of the source in two and three dimensions.

\begin{tabular}{lccc}
\hline \hline$d=2$ & $p=1$ & $p=0$ & $p=-1$ \\
\hline$\gamma=0$ & 1 & $\mathrm{Pe} /(\log \mathrm{Pe})^{1 / 2}$ & $\mathrm{Pe}$ \\
$0<\gamma<1$ & $\mathrm{Pe}^{\gamma}$ & $\mathrm{Pe}$ & $\mathrm{Pe}$ \\
$\gamma=1$ & $\mathrm{Pe} /(\log \mathrm{Pe})^{1 / 2}$ & $\mathrm{Pe}$ & $\mathrm{Pe}$ \\
$\gamma>1$ & $\mathrm{Pe}$ & $\mathrm{Pe}$ & $\mathrm{Pe}$ \\
\hline$d=3$ & & & \\
\hline$\gamma=0$ & 1 & $\mathrm{Pe}^{1 / 2}$ & $\mathrm{Pe}$ \\
$0<\gamma<1 / 2$ & 1 & $\mathrm{Pe}^{\gamma+1 / 2}$ & $\mathrm{Pe}$ \\
$\gamma=1 / 2$ & 1 & $\mathrm{Pe} /(\log \mathrm{Pe})^{1 / 2}$ & $\mathrm{Pe}$ \\
$1 / 2<\gamma<3 / 2$ & $\mathrm{Pe} \gamma-1 / 2$ & $\mathrm{Pe}$ & $\mathrm{Pe}$ \\
$\gamma=3 / 2$ & $\mathrm{Pe} /(\log \mathrm{Pe})^{1 / 2}$ & $\mathrm{Pe}$ & $\mathrm{Pe}$ \\
$\gamma>3 / 2$ & $\mathrm{Pe}$ & $\mathrm{Pe}$ & $\mathrm{Pe}$ \\
\hline \hline
\end{tabular}

pression is concerned - there can be then no "residual" effective diffusivity due to stirring in the limit of negligible molecular diffusion.

Acknowledgments. We are grateful for stimulating discussions with Francesco Paparella, Tiffany A. Shaw, William R. Young, and many participants of the 2005 GFD Program at Woods Hole Oceanographic Institution where much of this work was performed. CRD. was supported by NSF Grants Nos. PHY-0244859 and PHY-0555234 and the Alexander von Humboldt Foundation. J-LT was supported in part by the UK Engineering and Physical Sciences Research Council Grant GR/S72931/01.
[1] A.J. Majda \& P.R. Kramer, Phys. Rep. 314, 237 (1999); B.I. Shraiman \& E.D. Siggia, Nature 405, 639 (2000); B.L. Sawford, Annu. Rev. Fluid Mech. 33, 289 (2001); G. Falkovich, K. Gawędzki, \& M. Vergassola, Rev. Mod. Phys. 73, 913 (2001).

[2] B.L. Sawford \& J.C.R. Hunt, J. Fluid Mech. 165, 373 (1986).

[3] G. Mathew, I. Mezić, \& L. Petzold, Physica D 211, 23
(2005).

[4] J.-L. Thiffeault, C.R. Doering, \& J.D. Gibbon, J. Fluid Mech. 521, 105 (2004).

[5] S. Plasting \& W.R. Young, J. Fluid Mech. 552, 289 (2006).

[6] T.A. Shaw, J.-L. Thiffeault, \& C.R. Doering (2006), arXiv:physics/0607270. 